\title{
La Relación Centro Escolar-Familia como Factor Protector de Conductas Transgresoras en la Adolescencia
}

\author{
Ma José Rodrigo López $\left({ }^{*}\right)$, Raquel-Amaya Martínez González $\left({ }^{* *}\right)$ y Beatriz Rodríguez-Ruiz ${ }^{* *}$ ) \\ (*) Universidad de La Laguna y ${ }^{(*}$ ) Universidad de Oviedo
}

\begin{abstract}
RESUMEN
Este estudio se plantea con el objetivo de analizar la potencialidad predictiva de la relación entre los centros escolares y las familias sobre la actitud de los adolescentes hacia transgredir la autoridad institucional. La muestra está compuesta por 400 adolescentes de segundo y cuarto curso de educación secundaria obligatoria de Asturias y Santa Cruz de Tenerife (España), sus 400 padres y 400 madres y sus 125 profesores/as. Los datos se han recabado con el Cuestionario sobre la Colaboración Centro Escolar-Familia en sus versiones para padres y madres y para profesorado (Martínez-González, 1996) y con el Cuestionario de Actitud hacia la Autoridad Institucional para Adolescentes (Reicher y Emler, 1985; LISIS, 2005). Una vez efectuados análisis comparativos de grupos y predictivos, los resultados indican que las familias perciben una mayor implicación en los estudios de sus hijos y colaboración con el profesorado de la que perciben estos últimos; se constata también una mayor dedicación de las madres que de los padres. Los análisis de regresión señalan que cuando los padres y madres perciben una mayor implicación familiar en los estudios del hijo/a, se promueven en los/as adolescentes unas actitudes menos positivas hacia la transgresión de normas derivadas de la autoridad institucional, lo que permite conceptualizar dicha implicación como factor de protección de las actitudes transgresoras. Del estudio se derivan implicaciones para la práctica, como crear canales efectivos de comunicación entre los centros escolares y las familias y fomentar la implicación de la figura del padre además de la de la madre.
\end{abstract}

Palabras Clave: colaboración centro escolar-familia, autoridad institucional, adolescencia.

\section{The Family-School Relationship as a Protective Factor of Transgressive Behavior in Adolescence}

\section{ABSTRACT}

This study is aimed at analyzing the predictive potential of the relationship between schools and families on the attitude of adolescents to transgress institutional authority. The sample consists of 400 adolescents enrolled in the second and fourth year of compulsory secondary education in Asturias and Santa Cruz de Tenerife (Spain), their 400 fathers and 400 mothers and their 125 teachers. The data was collected with the School-Family Collaboration Questionnaire in its versions for fathers and mothers and for teachers (MartínezGonzález, 1996) and with the Questionnaire on Attitude towards the Institutional Authority for Adolescents (Reicher and Emler, 1985; LISIS, 2005). Once comparative groups and predictive analyzes were carried out, the results indicate that families perceive greater involvement in the studies of their children and collaboration with the teachers than the latter perceive on the same matters; there is also greater involvement on the part of the mothers than of the fathers. The regression analyzes indicate that when parents perceive a greater family involvement in the studies of the child, less positive attitudes towards the transgression of norms derived from the institutional authority are promoted in the adolescents, which allows to conceptualize this family involvement as a factor of protection from transgressive attitudes. From the study some conclusions and implications for the practice are derived.

Keywords: school-family collaboration, institutional authority, adolescence.

\section{Introducción}

La adolescencia es una etapa crítica del desarrollo donde además de los cambios que se producen a nivel personal, se pueden dar también alteraciones en las relaciones con el grupo familiar, con los amigos y con las instituciones sociales. Ello es comprensible ya que los/las adolescentes tienen que afrontar cambios en las interacciones personales e institucionales (Domínguez, López y Álvarez, 2015), dando lugar en ocasiones a actitudes de oposición ante las normas sociales, que pueden conllevar importantes problemas de disciplina ante la autoridad tanto parental como institucional. Ante esta situación, que afecta directamente a las familias y a los centros escolares (Elzo, 2006; Ridao y Moreno, 2008; López-Larrosa y Dubra, 2010), se espera que ambos contextos se impliquen y colaboren activamente para promover en los/as adolescentes una actitud positiva hacia las normas sociales. Por ello, este estudio tiene como objetivo analizar la percepción que tienen las familias y el profesorado 
sobre los procesos y la calidad de la relación que se establece entre ellos en función de variables sociodemográficas, y su posible influencia en la actitud que los/as adolescentes manifiestan ante la autoridad institucional.

\section{Relación entre el Centro Escolar y la Familia}

En la etapa de la adolescencia, el centro escolar y la familia siguen siendo los principales contextos de socialización, junto con el grupo de iguales, los medios de comunicación y las redes sociales. Numerosos autores han puesto de manifiesto la necesidad de promover la colaboración entre los padres y madres y el profesorado para responder de manera positiva a los continuos cambios que tienen lugar tanto en la institución familiar, que va modificando su estructura y sus dinámicas (Rodrigo et al., 2015), como en los centros escolares, que necesitan reorganizarse para poder dar una respuesta educativa de calidad a las nuevas necesidades sociales (Albaladejo, 2011; Gómez, 2006; Martínez-González, Rodríguez-Ruiz y Jimeno, 2010). Una alianza que no siempre se lleva a cabo con la frecuencia o calidad deseada, a pesar de que su necesidad se recoge en la legislación educativa como elemento de calidad educativa (Sarramona i López y Rodríguez Neira, 2010). En esta legislación se señala también el derecho de las familias a participar activamente con los centros escolares en el proceso educativo y académico de sus hijos (Frías del Val, 2014, Ley Orgánica 8/2013, de 9 de diciembre, para la Mejora de la Calidad Educativa; Vallespir, Rincón y Morey, 2016).

Los estudios realizados sobre la perspectiva de las familias acerca de su colaboración con los centros escolares indican que ésta es más frecuente en las etapas de la Educación Infantil y Educación Primaria, que en la etapa de la Educación Secundaria (Castro y García-Ruiz, 2013; Rivera y Milicic, 2006). En las primeras, se implican de manera más frecuente las madres (Nord, Brimhall y West, 1997; Valdés, Martín y Sánchez, 2009), dado que son también las protagonistas habituales de la crianza y educación de sus hijos/as (Pacheco, Rodríguez Díaz, y García Pérez, 2013), y las familias con niveles educativos superiores (MECD, 2014). Por su parte, el profesorado comparte la perspectiva de las familias sobre una mayor participación en las primeras etapas escolares, si bien indican que no es tan frecuente como desearían (Batle, Hernández y Mir, 2009; Castro y GarcíaRuiz, 2013; MECD, 2014); refieren la asistencia a reuniones convocadas por el profesorado o a actividades lúdicas deportivas o culturales (García-Bacete, 2006; Feito, 2014). En la etapa de secundaria los contactos entre ambos suelen estar vinculados a problemas de rendimiento o de comportamiento de los/as hijos/as (Giró, Mata, Vallespir, y Vigo, 2014) y, en ocasiones, el profesorado se percibe a sí mismo como un elemento que limita la participación de las familias en el centro escolar (Consejo Escolar del Estado, 2015).

Las investigaciones señalan los efectos positivos que genera la colaboración entre los centros escolares y las familias en los adolescentes; pueden mejorar su rendimiento académico (Martínez-González, 1996) y también en las esferas social y personal (Blanco y Martínez-González, 2017; Gârțu, 2017). Por su parte, las familias valoran de manera más positiva al profesorado, se implican más en el seguimiento de las tareas escolares de sus hijos (Lozano, 2003; Risko y Walker-Dalhouse, 2009), y se sienten más competentes en el desarrollo de su rol parental. En el caso del profesorado, percibe que puede desempeñar mejor su labor tutorial con el alumnado para contribuir a su desarrollo académico y personal, además de considerar a la familia un "aliado" para apoyar su proceso socializador (Jeynes, 2007;
Sheldon y Epstein, 2005). Carter y Reardon, (2014) sostienen que cuando se da dicha colaboración, los/as adolescentes con mayores dificultades sociales y económicas mejoran su asistencia al centro y sus resultados académicos y respetan más a la autoridad institucional. Así pues, se puede considerar que la colaboración centro escolar-familia constituye un factor de protección al promover modelos de conducta positivos (Williams y Bryan, 2013; Williams y Portman, 2014; Zalaquett y López, 2006).

\section{Actitud de los Adolescentes hacia la autoridad institucional}

Las interacciones sociales que los/as adolescentes mantienen en sus contextos más próximos de desarrollo, es decir, con sus padres, hermanos, amigos y profesorado, van cambiando de forma progresiva según avanzan los años, pasando de unas relaciones más asimétricas, controladas por la autoridad adulta, a unas relaciones más simétricas en las que se desempeña un rol más igualitario (Rodrigo, García, Máiquez, Rodríguez y Padrón, 2008). Estos cambios, en ocasiones se reflejan en diferentes puntos de vista, roces o enfrentamientos, que provocan un cuestionamiento de la autoridad (Rodríguez-Ruiz, 2012). Como cualquier transición vital, la adolescencia puede conllevar inestabilidad emocional, dificultades y riesgos de conductas inadaptadas, aunque también existan otras características positivas_(Oliva y Parra, 2004).

La vulnerabilidad de esta etapa puede producir episodios de violencia, en especial dentro del contexto familiar y escolar, con conductas transgresoras de las normas escolares y sociales (Cava, Musitu y Murgui, 2006), y el cuestionamiento de su importancia (Smith, 2016; Vega-Gea, Ortega-Ruiz y Sánchez, 2016), tal como reflejan numerosas investigaciones (Defensor del Menor, 2007; Olweus, 1998; Ortega y Mora-Merchán, 2000; Smith y Brain, 2000; Trianes, 2000). Estas conductas violentas de los/as adolescentes han sido relacionadas con sus actitudes negativas hacia la autoridad institucional (Estévez, Jiménez y Moreno, 2011; Musitu, Estévez y Emler, 2007), vinculando la transgresión de normas escolares con las actitudes positivas hacia la transgresión de otras normas sociales (Emler, Ohana y Moscovici, 1987; Estévez y Emler, 2009).

Diversas investigaciones han señalado que la actitud negativa de los/as adolescentes hacia la autoridad institucional se relaciona con conductas de riesgo, como consumo de sustancias o acciones delictivas (Emler y Reicher, 2005; Estévez y Emler, 2009), afectando a su desarrollo académico y con ello, a su futuro profesional y personal. Por el contrario, una actitud positiva hacia las normas sociales se asocia al bienestar subjetivo (Moncher y Miller, 1999). Es claro que el contexto familiar desempeña una función fundamental en promover el ajuste psicosocial en la adolescencia (Dekovic, Wissink y Meijer, 2004; Oliva, Parra, y Reina, 2014; Ruiz-Juan y Ruiz-Risueño, 2011), pero también el apoyo del profesorado y del grupo de iguales tienen un papel relevante en fomentar la satisfacción vital en esta etapa (Diener y Fujita, 1995). En la medida en que en ambos contextos no se fomente el respecto a la figura parental o del profesorado se favorecerán acciones más transgresoras en los/as adolescentes, potenciando una imagen social basada en la rebeldía y el inconformismo (Estévez, Moreno, Jiménez y Musitu, 2013).

Así pues, centro escolar y familia, así como las relaciones que se establecen entre ellos, desempeñan un papel relevante como factores de protección en la etapa de la adolescencia (Burt, Resnick y Novick, 1998; Emler, 2009; Huebner et al., 2004). A pesar de ello, existen pocos estudios que constaten el grado de influencia o cuáles son los aspectos de la relación 
centro escolar-familia que mejor predicen las actitudes positivas hacia esta autoridad institucional (Molpeceres, Lucas y Pons, 2000). El presente estudio examina qué factores de la relación entre el centro escolar y la familia influyen en la promoción de dichas actitudes, ya sean de aceptación de dicha autoridad o de transgresión. Para ello, se plantean como objetivos analizar la percepción tanto de los padres y madres como del profesorado sobre sus relaciones mutuas, así como las actitudes del alumnado adolescente hacia la autoridad institucional. Posteriormente se pretende identificar el grado en que las relaciones entre los centros escolares y las familias percibidas por ambos colectivos pueden contribuir a explicar parte de dicha actitud del alumnado.

\section{Método}

\section{Participantes}

El total de participantes asciende a 1445, procedentes de las provincias de Asturias y de Santa Cruz de Tenerife (España); se encuentran distribuidos en cuatro grupos: 440 padres y 440 madres con hijos/as adolescentes, sus 440 hijos/as que cursan $2^{\circ}$ y $4^{\circ}$ de Enseñanza Secundaria Obligatoria (ESO), y 125 profesores/ as que imparten docencia a estos/as adolescentes.

Los padres y madres (ver Tabla 1) están mayoritariamente casado/as (92\%), con edades entre 30 y 62 años, con una media de 45.51 años el padre (DT $=5.47$ ) y 43.29 años la madre (DT =5.22), confirmando la tendencia nacional de que las madres son más jóvenes que los progenitores varones (INE, 2005a). El nivel educativo de los padres y madres mayoritariamente es de bachillerato o formación profesional, su situación laboral mayoritaria es de ocupados/as y un $25.9 \%$ de las madres trabaja de manera exclusiva y no remunerada en el hogar familiar.

Tabla 1. Variables sociodemográficas del padre y la madre.

\begin{tabular}{lcc}
\hline & $\begin{array}{c}\text { Padre } \\
\text { M (DT) o \% }\end{array}$ & $\begin{array}{c}\text { Madre } \\
\text { M (DT) o \% }\end{array}$ \\
\hline Edad & $45.51(5.47)$ & $43.29(5.22)$ \\
Estado civil & 92.7 & 92.0 \\
Casado/a & 5.9 & 6.2 \\
Divorciado/a-Separado/a & 0.5 & 0.9 \\
Soltero/a & 0.9 & 0.9 \\
Pareja de hecho & & 27.0 \\
Nivel de estudios & 33.4 & 41.0 \\
Nivel bajo: graduado escolar & 38.9 & 32.0 \\
Nivel medio: bachillerato y FP & 27.7 & 67.5 \\
Nivel alto: universitarios & & 5.0 \\
Situación laboral & 92.5 & 1.6 \\
Ocupado/a & 2.3 & 25.9 \\
Parado/a & 5.2 & \\
Jubilado/a-Pensionista & 0.0 & \\
Ama de casa & &
\end{tabular}

Respecto a los/as adolescentes (ver Tabla 2), el 49.2\% son chicas; el $57.7 \%$ cursan $2^{\circ}$ de la ESO y el $42.3 \%$, $4^{\circ}$ de este nivel, con una edad media de 14.1 años (Media $2^{\circ} \mathrm{ESO}=13.3 \mathrm{años}$; Media $4^{\circ} \mathrm{ESO}=15.2$ años). La tipología familiar de la mayoría es biparental (93\%); respecto al número de hermanos, el 51.5\% tienen uno, situación que se ajusta a la tendencia nacional de las familias españolas (INE, 2017). Finalmente, considerando el tipo de centro escolar, asisten sobre todo a centros públicos (39.1\%) y concertados $(38.6 \%)$, teniendo porcentajes más bajos los centros privados $(22.3 \%)$.
Tabla 2. Variables sociodemográficas de los adolescentes.

\begin{tabular}{lc}
\hline Género & Adolescente $(\%)$ \\
\hline Masculino & 50.8 \\
Femenino & 49.2 \\
Curso escolar & \\
$2^{\mathbf{o}}$ ESO & 57.7 \\
$4^{\underline{0}}$ ESO & 42.3 \\
Tipología familiar & \\
Biparental & 93.0 \\
Monoparental & 7.0 \\
Número de hermanos & \\
Ningún hermano & 28.2 \\
Uno & 51.5 \\
Dos & 14.5 \\
Tres o más & 5.8 \\
Tipología de centro escolar & \\
Público & 39.1 \\
Privado & 22.3 \\
Concertado & 38.6 \\
\hline
\end{tabular}

El profesorado imparte docencia en $2^{\circ}$ y $4^{\circ}$ de la ESO a los hijos/as de las familias descritas con anterioridad. En la tabla 3 se observa la distribución en función del género (60\% profesoras), con una edad media mayoritaria de 43.63 años (intervalo 4052 años, $45.6 \%$ ). Con relación a la materia escolar impartida, en el $54.4 \%$ de los casos es de Letras (lengua española, inglés o filosofía) y en el $45.6 \%$ restante es de Ciencias (física, química o matemáticas). Respecto a la experiencia docente, un $74.4 \%$ cuenta entre 1-22 años.

Tabla 3. Variables sociodemográficas del profesorado.

\begin{tabular}{lc}
\hline Género & Profesorado (\%) \\
\hline Masculino & 40.0 \\
Femenino & 60.0 \\
Edad & \\
27-39 años & 36.0 \\
40-52 años & 45.6 \\
53-65 años & 18.4 \\
Especialidad & \\
Ciencias & 45.6 \\
Letras & 54.4 \\
Experiencia docente & \\
1-11 años & 30.4 \\
12-22 años & 44.0 \\
23-33 años & 17.6 \\
34-44 años & 8.0 \\
\hline
\end{tabular}

\section{Instrumentos}

- Cuestionario sobre la Colaboración Centro Escolar-Familia en sus versiones para padres y madres y para profesorado (Martínez-González, 1996). Consta de 58 ítems con respuesta en escala de Likert, analizando, por una parte, frecuencia (1-nunca; 2-pocas veces; 3-algunas veces; 4-a menudo y 5-siempre) y, por otra, actitudes (1-nada de acuerdo; 2-poco de acuerdo; 3-moderadamente de acuerdo; 4-muy de acuerdo y 5-totalmente de acuerdo). En una investigación previa con la muestra de este estudio (Rodríguez-Ruiz, 2012; Rodríguez Ruiz et al., 2016), la versión para la familia se estructuró en 5 factores con una varianza explicada del $57.40 \%$ : Factor 1-Percepción de la familia sobre la colaboración del profesorado (19 ítems, $\alpha=.94$ ); analiza las valoraciones 
del padre y de la madre sobre: 1) la capacitación del profesorado de sus hijos/as para el desarrollo de su labor docente, 2) su colaboración con el profesorado de sus hijos/as en el proceso de educación y socialización, y 3) la ayuda y colaboración que reciben del profesorado de sus hijos/as para resolver problemas personales, familiares y escolares; Factor 2-Contactos entre la familia y el profesorado (8 ítems, $\alpha=.84$ ); analiza las relaciones, reuniones $\mathrm{y}$ contactos directos entre la familia y el profesorado de sus hijos/as por iniciativa tanto de la familia o del propio profesorado, con la finalidad de profundizar en el desarrollo académico de los hijos/as; Factor 3-Estudios del hijola e implicación de la familia (10 ítems, $\alpha=.72$ ); analiza las actividades de supervisión, apoyo y ayuda que los padres y las madres desarrollan con relación a los estudios de sus hijos; Factor 4-Relación con el centro escolar (11 ítems, $\alpha$.69), analiza las relaciones no cooperativas que los padres y las madres establecen con el centro escolar y los motivos por los cuáles no participan en el mismo y Factor 5-Apoyo familiar en los estudios y participación de los padres en el centro (10 ítems, $\alpha=.72$ ), analiza el asesoramiento que las familias reciben del centro escolar así como su participación en las actividades lúdicas, académicas y culturales que organiza el centro.

- Laversión parael profesorado obtuvo una estructura factorial de 4 factores con una varianza explicada del 73.03\%: Factor 1-Percepción del profesorado sobre la implicación de la familia en la educación de los hijos/as (31 ítems, $\alpha=.98$ ); analiza las valoraciones que realiza el profesorado sobre: 1) las competencias de los padres y madres para desarrollar su rol parental, 2) la colaboración con las familias de sus alumnos/as en el proceso de educación y socialización y 3) la ayuda y colaboración de las familias de su alumnado en la resolución de problemas personales, familiares y escolares; Factor 2-Contactos entre el profesorado y la familia (13 ítems, $\alpha=.92)$; analiza las relaciones, reuniones y contactos directos entre el profesorado y la familia por iniciativa del profesorado y /o por iniciativa de la propia familia, con la finalidad de profundizar en el desarrollo académico del alumnado; Factor 3-Estudios del hijo e implicación de la familia (6 ítems, $\alpha=.83$ ); analiza la percepción del profesorado sobre las actividades de supervisión, el apoyo y la ayuda que los padres y las madres desarrollan en relación con los estudios de sus hijos/as, y Factor 4-Apoyo y ayuda de los centros escolares a las familias (4 ítems, $\alpha=.82$ ); evalúa el apoyo, el asesoramiento y la ayuda que reciben las familias del centro escolar.

- Cuestionario de Actitud hacia la Autoridad Institucional para Adolescentes (Reicher y Emler, 1985; versión adaptada por el Equipo LISIS de la Universidad de Valencia, 2005). Valora la actitud de los/as adolescentes hacia la autoridad institucional respecto a diferentes contextos sociales. Consta de 10 ítems con respuesta tipo Likert de grado de acuerdo de cinco alternativas (1-nada de acuerdo; 2-poco de acuerdo; 3-moderadamente de acuerdo; 4-muy de acuerdo y 5 -totalmente de acuerdo). La estructura factorial obtenida en el instrumento se corresponde con la versión original de los autores y cuenta con dos factores: Factor 1-Actitud positiva hacia la autoridad (6 ítems, $\alpha=.73$ ), que explica el $26.43 \%$ de la varianza y Factor 2-Actitud positiva hacia la transgresión de normas sociales (4 ítems, $\alpha=80$ ), que explica el $25.47 \%$ de la varianza.

\section{Procedimiento}

Se contactó por carta con 26 centros escolares de las provincias mencionadas. El 50\% aceptaron participar, aunque solamente 12 se han incluido en este estudio por contar solo en ellos con información a la vez para un mismo alumno/a tanto del padre y de la madre, como del profesorado y del propio alumno/a.

Cuando los centros escolares aceptaron participar, se envió a todas las familias del alumnado de $2^{\underline{0}}$ y $4^{\underline{0}}$ de la ESO una carta en la que se solicitaba su participación, así como cumplimentar un protocolo a través del cual daban su consentimiento, garantizando el anonimato de la información suministrada. Se obtuvieron autorizaciones de las familias en un 95\% de los casos, si bien no todas ellas cubrieron finalmente la escala a aplicar. El porcentaje final recogido resultó del 85\%. La escala para las familias se entregó al alumnado dentro de un sobre que incluía instrucciones muy detalladas sobre cómo cumplimentarla.

Al mismo tiempo, se entregó al profesorado que impartía docencia en esos cursos el instrumento de recogida de información. Por otra parte, un miembro del equipo investigador se encargó de la recogida de información de los/as adolescentes en los propios centros. El procedimiento fue aprobado por el Comité de Ética de la Investigación y de Bienestar Animal (CEIBA) de la Universidad de La Laguna.

\section{Análisis de datos}

Los análisis realizados han consistido en: 1) Análisis descriptivos: frecuencias, medidas de tendencia central (Media, M) y variabilidad (desviación típica, DT); 2) Contrastes de dos grupos para muestras independientes a través de la t de Student, entre los factores analizados y las variables sociodemográficas género en los progenitores y en el profesorado, y curso escolar en los adolescentes. Se ha calculado el tamaño del efecto con la d de Cohen (1969) cuando las diferencias resultaron significativas; 3) Contrastes de tres grupos mediante ANOVA de un factor con los factores de estudio y el nivel educativo para las familias y edad para el profesorado; la selección de estas variables se ha realizado teniendo en cuenta que pueden incidir de manera diferencial en los aspectos analizados (Armas, 2012; Castro y García-Ruiz, 2013). En cada análisis se tuvo en cuenta si se cumplía la condición de homogeneidad de las varianzas (prueba de Levene). Cuando se cumplía esta condición y cuando las diferencias resultaron significativas se realizaron contrastes a posteriori de dos grupos utilizando el método de Scheffé. En los casos en los que no se cumplía la condición de homogeneidad de varianzas se recurrió al estadístico de Welch, como una alternativa robusta. Para valorar el tamaño del efecto de las diferencias entre grupos se utilizó el estadístico $\mathrm{R}^{2}$. Siguiendo las recomendaciones de Cohen (1969), se considera muy bajo cuando $\mathrm{R}^{2}<.01$, bajo cuando $.01<\mathrm{R}^{2}<.09$, medio cuando $.09<\mathrm{R}^{2}<.25$ y alto cuando $\mathrm{R}^{2}>.25$; y 4 ) Análisis de regresión lineal múltiple jerárquico en tres pasos con la finalidad de realizar predicciones sobre la actitud ante la autoridad institucional de los adolescentes en función de la relación centro escolar-familia desde la perspectiva de las familias y del profesorado. Para ello se exploraron inicialmente los supuestos de linealidad, normalidad y homocedasticidad. En el primer paso se controlaron variables sociodemográficas de los adolescentes (género y curso escolar), en el segundo paso se incluyeron los factores de relación centro escolar y familia valorada por las familias, y finalmente, en el tercer paso se incluyeron las variables de relación centro escolar y familia valorada por el profesorado. Para interpretar la significación global del modelo se examinó el estadístico F, 
así como los valores de la $\mathrm{R}$ y $\mathrm{R}^{2}$ de cambio. Además, se observó la contribución específica de cada dimensión a la varianza total explicada por el modelo a través de la significatividad y el valor de la correlación semiparcial al cuadrado $\left(\mathrm{rs}^{2}\right)$.

\section{Resultados}

\section{a) Análisis descriptivos y comparativos}

Percepción de la Relación entre el Centro Escolar y la Familia desde la perspectiva de los Padres y Madres

Los análisis descriptivos indican que tanto los padres como las madres perciben una alta implicación en la educación de sus hijos/as en casa (Factor 3, Padre $\mathrm{M}=4.08$; DT $=.44$ - Madre $\mathrm{M}=4.10 ; \mathrm{DT}=.44)$ y que el profesorado colabora con ellos/ as (Factor 1, Padre $\mathrm{M}=3.72$; DT = .75 - Madre $\mathrm{M}=3.78$; DT = .68). Sin embargo, ambos manifiestan que son poco frecuentes los contactos de la familia con el profesorado (Factor 2, Padre $\mathrm{M}=2.46 ; \mathrm{DT}=.96-$ Madre $\mathrm{M}=2.47 ; \mathrm{DT}=.90)$, la relación con el centro escolar (Factor 4, Padre $\mathrm{M}=2.16$; $\mathrm{DT}=.52-$ Madre $\mathrm{M}=$ 2.13; DT = .50) y el apoyo familiar en el estudio y la participación escolar (Factor 5, Padre $\mathrm{M}=2.93$; $\mathrm{DT}=.68$ - Madre $\mathrm{M}=3.08$; $\mathrm{DT}=$ .61). Los análisis comparativos en función del género (ver Tabla 4) encuentran diferencias significativas en el Factor 1-Percepción sobre la colaboración del profesorado, indicando que las madres perciben mayor colaboración del profesorado que los padres, $t$ $(438)=-2.26 ; p=.024)(d$ Cohen= -0.08). Respecto al Factor 5-Apoyo familiar en los estudios y participación de la familia en el centro escolar $(t(439)=-5.58 ; p=.000)(d$ Cohen $=-0.23)$ los análisis muestran que el padre apoya a sus hijos/as en los estudios con menos frecuencia que la madre, y participa menos que ellas en el centro escolar.

Tabla 4. Diferencias significativas en la Percepción de la Relación entre el Centro Escolar y la Familia desde la perspectiva de los Padres y Madres.

\begin{tabular}{|c|c|c|c|c|c|}
\hline & & M (DT) & $\mathbf{t}$ & $\mathbf{p}$ & d Cohen \\
\hline \multicolumn{6}{|l|}{ En función del género } \\
\hline \multirow[t]{2}{*}{ Factor 1-Percepción de las familias sobre la colaboración del profesorado } & Padre & $3.72(.75)$ & -2.26 & .024 & -0.08 \\
\hline & Madre & $3.78(.68)$ & & & \\
\hline \multirow[t]{3}{*}{ Factor 5-Apoyo familiar en el estudio y la participación de la familia en el centro escolar } & Padre & $2.93(.68)$ & -5.58 & .000 & -0.23 \\
\hline & Madre & $3.08(.61)$ & & & \\
\hline & & M (DT) & $\mathbf{F}$ & p & $\mathbf{R}^{2}$ \\
\hline \multicolumn{6}{|l|}{ En función del nivel educativo } \\
\hline Factor 3-Percepción de la familia sobre los estudios del hijo/a e implicación de la familia & Padre & $4.08(.44)$ & 3.636 & .027 & 0.16 \\
\hline Factor 4-Relación con el centro escolar & & $2.16(.52)$ & 3.046 & .029 & .014 \\
\hline Factor 3-Percepción de la familia sobre los estudios del hijo/a e implicación de la familia & Madre & $4.10(.44)$ & 4.270 & .015 & .019 \\
\hline
\end{tabular}

Tomando en consideración la figura del padre, el análisis comparativo en función del nivel de estudios muestra diferencias en el Factor 3-Percepción de la familia sobre los estudios del hijo/a e implicación de la familia $(F(2,437)=3.636 ; p=.027)$ con un tamaño del efecto bajo $\left(R^{2}=0.16\right)$. Los padres con estudios universitarios perciben que se implican significativamente con mayor frecuencia en los estudios de sus hijos/as $(\mathrm{M}=4.17$; $\mathrm{DT}=.37)$ que quienes tienen estudios primarios $(\mathrm{M}=4.03$; $\mathrm{DT}=.48)(\mathrm{DFS}=0.13$, $\mathrm{p}=.037)$. Respecto al Factor 4-Relación con el centro escolar, también existen diferencias significativas $(F(3,438)=3.046 ; p=.029)$ con un tamaño del efecto bajo $\left(R^{2}=0.14\right)$ : los padres con estudios primarios y graduado escolar apoyan significativamente con menor frecuencia los estudios de sus hijos/as y participan menos en el centro escolar $(\mathrm{M}=2.75$; $\mathrm{DT}=.52)$ que los padres con estudios de bachillerato y formación profesional $(\mathrm{M}=3.01$; $\mathrm{DT}=.35)(\mathrm{DFS}=0.26, \mathrm{p}=.003)$ y universitarios $(\mathrm{M}=3.20 ; \mathrm{DT}=.42)$ $(\mathrm{DFS}=0.30, \mathrm{p}=.001)$.

El análisis comparativo para la madre en función del nivel de estudios (tabla 5) señala también diferencias en el Factor 3Percepción de la familia sobre los estudios del hijo/a e implicación de la familia $(F(2,439)=4.270 ; p=.015)$ con un tamaño del efecto bajo $\left(R^{2}=0.19\right)$. En concreto, las madres con estudios primarios y graduado escolar se implican significativamente menos en los estudios de sus hijos/as $(\mathrm{M}=4.01$; $\mathrm{DT}=.45)$ que las madres con estudios universitarios $(\mathrm{M}=4.17$; $\mathrm{DT}=.39)(\mathrm{DFS}=0.15, \mathrm{p}=.015)$
Percepción de la Relación entre el Centro Escolar y la Familia desde la perspectiva del Profesorado

Los resultados descriptivos indican que el profesorado tiene una percepción moderadamente positiva tanto de la implicación de la familia en los estudios y la crianza de los hijos/as (Factor 1, M = 3.06; DT = .40), como de la implicación del centro escolar para ayudar a las familias (Factor 4, M=3.66; $\mathrm{DT}=.27)$, mostrando una percepción menos positiva respecto a la implicación de las familias en las tareas escolares (Factor 1, $\mathrm{M}=2.65 ; \mathrm{DT}=.33$ ) y de los contactos con el profesorado (Factor $2, \mathrm{M}=2.47$; DT $=.84$ ).

Los análisis comparativos para la variable género (ver Tabla 5) señalan diferencias significativas en la percepción que tiene el profesorado sobre el Factor 2-Percepción del profesorado sobre los contactos con las familias $(t(101)=-3.38 ; p=.001)$ ( $d$ Cohen= -0.15). En concreto, las profesoras $(\mathrm{M}=2.77$; $\mathrm{DT}=.40)$ perciben significativamente más contactos que los profesores $(\mathrm{M}=2.64$; $\mathrm{DT}=.19)$. En la variable edad también se obtienen diferencias significativas $(F(2,437)=3.492 ; p=.031)$ con un tamaño del efecto bajo $\left(R^{2}=.016\right)$, indicando que el profesorado con edades comprendidas entre 31 y 39 años percibe menos contactos $(\mathrm{M}=$ 2.56; DT =.25) que quienes tienen entre 49 y 57 años $(\mathrm{M}=2.96$; DT $=.56)(\mathrm{DFS}=0.40, \mathrm{p}=.000)$.

Finalmente, respecto a la percepción que tiene el profesorado sobre el Factor 1- Percepción del profesorado sobre la implicación de la familia en la educación de los hijos/as, se observan diferencias en 
Tabla 5. Diferencias significativas en la Percepción de la Relación entre el Centro Escolar y la Familia desde la perspectiva del Profesorado.

M (DT)

$\mathbf{t}$

p d Cohen

En función del género

Factor 2-Percepción del profesorado sobre los contactos con las familias

Profesor

$2.64(.19)$

$-3.38$

.001

$-0.15$

Profesora

$2.77(.40)$

F

p

$\mathbf{R}^{2}$

En función de la edad

Factor 2- Percepción del profesorado sobre los contactos con las familias

31-39 años

49-57 años

Factor 1- Percepción del profesorado sobre la implicación de la familia

en la educación de los hijos/as
31-39 años

40-48 años

49-57 años
M (DT)

$2.37(.93)$
$2.71(.79)$
$2.90(.26)$
$3.16(.40)$
$3.08(.51)$

3.492

.031

.016

22.174

.000

.092 la variable edad $(F 2,437)=22.171 ; p=.000)$ con un tamaño del efecto alto $\left(R^{2}=.092\right)$. En concreto, el profesorado con edades comprendidas entre 31 y 39 años percibe menos implicación (M = 2.90; $\mathrm{DT}=.26)$ que el que tiene entre 40 y 48 años $(\mathrm{M}=3.16$; DT $=.40)(\mathrm{DFS}=-0.26, \mathrm{p}=.000)$ y que el que tiene entre $49-57$ años $(\mathrm{M}$ $=3.08 ; \mathrm{DT}=.51)(\mathrm{DFS}=-0.18, \mathrm{p}=.008)$.

\section{Actitud de los Adolescentes hacia la autoridad institucional}

Los resultados obtenidos (ver Tabla 6) indican que los/as adolescentes muestran una actitud positiva hacia la autoridad institucional (Factor 1) $(\mathrm{M}=3.35, \mathrm{DT}=0.77)$, y están poco de acuerdo con la transgresión de normas sociales (Factor 2) $(\mathrm{M}=2.10, \mathrm{DT}=0.95)$. Con respecto a esta última, se han encontrado diferencias significativas en función del curso escolar ( $t$ (437) = 2.26; $p=.024)(d$ Cohen= 0.22); en concreto, los/as adolescentes que cursan $2^{\circ}$ de la ESO aceptan más dicha transgresión $(\mathrm{M}=$ 2.19; DT =1.02) que sus compañeros/as de $4^{\circ}$ de la ESO $(\mathrm{M}=$ $1.98 ; \mathrm{DT}=.82$ ).

Tabla 6. Diferencias estadísticamente significativas en la Actitud de los/as Adolescentes hacia la Autoridad Institucional

\begin{tabular}{|c|c|c|c|c|c|}
\hline & & M (DT) & $\mathbf{t}$ & p & d Cohen \\
\hline \multicolumn{6}{|l|}{ En función del curso escolar } \\
\hline Factor 2-Actitud positiva hacia la transgresión de normas sociales & $\begin{array}{l}2^{\circ} \mathrm{ESO} \\
4^{\mathrm{o}} \mathrm{ESO}\end{array}$ & $\begin{array}{c}2.19(1.02) \\
1.98(.82)\end{array}$ & 2.26 & .024 & 0.22 \\
\hline
\end{tabular}

\section{b) Modelo Predictivo de la Relación Centro Escolar-Familia sobre la Actitud de los/as Adolescentes ante la Autoridad Institucional}

Con respecto a los análisis de regresión efectuados sobre los dos factores que valoran la actitud de los/as adolescentes ante la autoridad institucional, en el Factor 1-Actitud positiva hacia la autoridad institucional (ver Tabla 7), el modelo de regresión resultó significativo $(F(3,367)=6.39, p=.000)$, con un $R^{2}=.338$ ( $34 \%$ de la varianza explicada) en tres pasos, con contribuciones más relevantes para los pasos 2 y 3 que para las variables sociodemográficas introducidas en el paso 1. Así, se obtuvo un $\mathrm{R}^{2}$ cambio de .091 en el paso 2 (valoración de la familia de la colaboración centro escolar-familia) y un $\mathrm{R}^{2}$ cambio de .014 en el paso 3 (valoración del profesorado de la colaboración centro escolar-familia).

Los factores de la Relación entre Centro Escolar-Familia que contribuyeron de forma significativa al modelo fueron: Factor 1-Percepción de las familias sobre la colaboración del profesorado ( $\beta$ $=.15, p=.017)$, indicando que cuanto mejor es esta percepción, más positiva es la actitud de los adolescentes hacia la autoridad institucional; Factor 4-Percepción del Profesorado sobre la implicación del centro escolar para ayudar a las familias $(\beta=.15, p=.004)$ indicando también que cuanto mejor percibe el profesorado que el centro escolar ayuda a las familias, los/as adolecentes tienden a tener mejor actitud hacia la autoridad, y el Factor 3-Percepción del Profesorado sobre los estudios del hijo/a e implicación de la familia $(\beta=-.12, \quad p=.020)$, indicando una relación inversa: cuanto más percibe el profesorado que se implica la familia en dichos estudios menos positiva es la actitud de los/as adolescentes hacia la autoridad institucional.

Un análisis de los coeficientes de correlación semiparcial al cuadrado indica que cada uno de estos factores realizó una contribución individual al modelo, con mayor peso de las variables relativas a la implicación del centro escolar percibida por el profesorado para ayudar a las familias (Factor 4) $\left(\mathrm{rs}^{2}\right.$ $=$.14), la percepción de las familias sobre la colaboración del profesorado (Factor 1$)\left(\mathrm{rs}^{2}=.12\right)$ y la percepción del profesorado sobre los estudios del hijo/a e implicación familiar (Factor 3) $\left(\mathrm{rs}^{2}\right.$ $=-.11$ ).

Con relación al Factor 2-Actitud positiva de los/as adolescentes hacia la trasgresión de las normas sociales (tabla 8), el modelo de 
Tabla 7. Modelo predictivo para el paso 3 de la Relación Centro Escolar-Familia según la Familia y el Profesorado sobre la Actitud Positiva de los/as Adolescentes hacia la Autoridad Institucional (Factor 1).

\begin{tabular}{|c|c|c|c|c|c|c|}
\hline \multicolumn{2}{|c|}{ B estandarizada } & \multirow{2}{*}{$\begin{array}{c}R^{2} \\
.225\end{array}$} & \multirow{2}{*}{$\frac{R^{2} \text { cambio }}{.014}$} & \multirow{2}{*}{$\frac{g l}{2 / 368}$} & \multirow{2}{*}{$\frac{F}{5.614}$} & \multirow{2}{*}{$\begin{array}{c}p \\
.010\end{array}$} \\
\hline Factores Relación Centro-Familia Predictores Actitud Positiva Adolescente & & & & & & \\
\hline Sexo & .050 & & & & & .315 \\
\hline Curso Escolar & -.058 & & & & & .257 \\
\hline Factor 1-Percepción de las familias sobre colaboración del profesorado & .153 & & & & & .017 \\
\hline Factor 2-Contactos entre la familia y el profesorado & .018 & & & & & .749 \\
\hline Factor 3-Percepción de la familia sobre los estudios del hijo/a e implicación familiar & .029 & & & & & .619 \\
\hline Factor 4-Relación con el centro escolar & .004 & & & & & .942 \\
\hline Factor 5-Apoyo familiar en los estudios y participación de los padres en el centro & -.106 & & & & & .067 \\
\hline Factor 1- Percepción del profesorado sobre la implicación de la familia en la educación de los hijos/as & .015 & & & & & .809 \\
\hline Factor 2-Percepción del Profesorado sobre los contactos con las familias & -.016 & & & & & .766 \\
\hline Factor 3- Percepción del Profesorado sobre los estudios del hijola e implicación de la familia & -.124 & & & & & .020 \\
\hline Factor 4- Percepción del profesorado sobre el apoyo y ayuda de los centros escolares a las familias & .155 & & & & & .004 \\
\hline
\end{tabular}

regresión resultó significativo en dos pasos $(F 3,367)=6.39$, $p=.000)$, con un $R^{2}=.225$ ( $22 \%$ de la varianza explicada), no realizando contribuciones los factores asociados al profesorado. Así, se obtuvo un $\mathrm{R}^{2}$ cambio de 0.11 en el paso 2, en el que el Factor 3-Percepción de la familia sobre los estudios del hijo/a e implicación familiar $(\beta=-.26 p=.020)$ contribuyó de forma significativa indicando una relación inversa: cuanto menos perciben las familias que se implican en los estudios de sus hijos/as, más tienden éstos a trasgredir las normas sociales.

Tabla 8. Modelo predictivo para el paso 2 de la Relación Centro Escolar-Familia según la Familia sobre la Actitud Positiva de lo/as Adolescentes hacia la Transgresión de las Normas Sociales (Factor 2).

\begin{tabular}{|c|c|c|c|c|c|c|}
\hline \multicolumn{2}{|c|}{ B estandarizada } & $R^{2}$ & $R^{2}$ cambio & $g l$ & $F$ & $p$ \\
\hline Factores Relación Centro-Familia Predictores Actitud Negativa Adolescente & & .225 & .011 & $2 / 368$ & 5.614 & .010 \\
\hline Sexo & -.050 & & & & & .337 \\
\hline Curso Escolar & -.231 & & & & & .015 \\
\hline Factor 1-Percepción de la familia sobre la colaboración del profesorado & -.056 & & & & & .337 \\
\hline Factor 2-Contactos entre la familia y el profesorado & -.098 & & & & & .059 \\
\hline Factor 3-Percepción de la familia sobre los estudios del hijo/a e implicación familiar & -.263 & & & & & .020 \\
\hline Factor 4-Relación con el centro escolar & .070 & & & & & .071 \\
\hline Factor 5-Apoyo familiar en los estudios y participación de los padres en el centro & -.082 & & & & & -.078 \\
\hline Factor 1- Percepción del profesorado sobre la implicación de la familia en la educación de los hijos/as & .045 & & & & & .383 \\
\hline Factor 2- Percepción del Profesorado sobre los contactos con las familias & -.015 & & & & & .769 \\
\hline Factor 3-Percepción del Profesorado sobre los estudios del hijo/ e implicación de la familia & -.015 & & & & & .774 \\
\hline Factor 4- Percepción del profesorado sobre el apoyo y ayuda de los centros escolares a las familias & -.078 & & & & & .138 \\
\hline
\end{tabular}

El análisis de los coeficientes de correlación semiparcial al cuadrado indica que el curso escolar contribuyó al modelo con una relación inversa $\left(\mathrm{rs}^{2}=-.12\right)$, indicando que a medida que avanza el curso y, por tanto, se incrementa la edad del alumnado ( $4^{\circ}$ de la ESO frente a $2^{\circ}$ ), los/as adolescentes tienden a tener una actitud menos transgresora de las normas sociales. Por su parte, el Factor 3-Percepción de la familia sobre los estudios del hijo e implicación familiar $\left(\mathrm{rs}^{2}=-.11\right)$ contribuyó también con relación inversa, como se indicó con anterioridad: a más percepción de implicación por parte de la familia, menos actitud positiva de los/as adolescentes a la transgresión.

\section{Discusión}

En el presente estudio se ha analizado la potencialidad predictiva de la relación centro escolar-familia sobre la actitud de los/as adolescentes hacia la autoridad institucional; para ello, se han identificado, desde la perspectiva de las familias y del profesorado, distintos factores que caracterizan la relación entre ambos contextos en función de las variables sociodemográficas. Los resultados señalan que las madres perciben más quelos padres la colaboración del profesorado, quizás porque tradicionalmente son ellas quienes se ocupan más de comunicarse con el centro. En el ámbito familiar, la colaboración de las familias se centra sustancialmente en intentar apoyar a los hijo/as en las tareas escolares (Musitu y Cava, 2001; Gârțu, 2017), principalmente por parte de las madres (Valdés et al., 2009). Algunas investigaciones señalan que las madres se implican de manera más activa en la educación de sus hijos/as (Pacheco et al., 2013) y en mantener una relación más frecuente con el profesorado (Rodríguez-

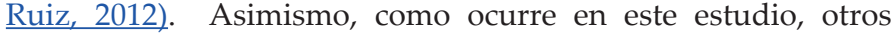
investigadores también constatan que las familias con niveles educativos superiores son quienes mantienen más contactos con el profesorado (Ministerio de Educación, Cultura y Deporte, 2014; Rivera y Milicic, 2006; Rodríguez-Ruiz, 2012). Este resultado puede indicar la relevancia que se concede al rendimiento escolar en las familias con niveles de estudios altos; estos resultados son coherentes con los encontrados en una investigación realizada por Martínez-González y Álvarez (2005) con alumnado de educación secundaria obligatoria en riesgo de abandono; se observó que este problema está más asociado a familias cuyos padres y madres tienen estudios de educación primaria.

Los resultados obtenidos respecto a la participación de las familias indican que, en la etapa de la educación secundaria 
obligatoria, al igual que en el resto de las etapas educativas, es la madre quien tiene un papel más activo, confirmando resultados obtenidos también en otras investigaciones (Blanco y MartínezGonzález, 2017; Pacheco et al., 2013). De estos estudios se deriva el rol relevante que desempeñan las madres para que sus hijos e hijas consigan un rendimiento académico adecuado que les facilite alcanzar éxito profesional en el futuro. Esto parece más claro aún en las madres con estudios universitarios, que se implican más y colaboran más directamente con el profesorado, percibiéndolo como un "aliado" en su rol educativo.

En el contexto escolar, la perspectiva del profesorado difiere de la de las familias, señalando una percepción menos positiva de esta implicación familiar en las tareas escolares de sus hijos y sobre la frecuencia de los contactos que mantiene con ellas (Batle et al. 2009; Castro y García-Ruiz, 2013). Además, de nuevo el género femenino, en este caso el de las profesoras, señala una perspectiva más positiva sobre la participación de las familias, señalando contactos más frecuentes con el profesorado (Andrés y Giró, 2016); una visión que aproxima las perspectivas de las profesoras y de las madres respecto a la relación entre los dos contextos.

Ambos entornos están participando conjuntamente del proceso socializador de los/as adolescentes, pero resulta necesario concretar cuáles pueden ser los aspectos, en uno y otro ámbito, que mejor predicen las actitudes hacia la autoridad institucional. Los resultados obtenidos en esta investigación sostienen que las percepciones de las familias sobre una mayor colaboración del profesorado promueven en los/as adolescentes unas actitudes más positivas hacia la autoridad institucional (Burt et al., 1998; Carter y Reardon, 2014). Así pues, cuando las familias perciben que ambos contextos van en la misma dirección, llegan a acuerdos, comparten sus experiencias y cooperan en la socialización de los/as adolescentes, se generan beneficios en la manera en que estos/as perciben las figuras de autoridad institucional. Asimismo, cuando los padres y madres perciben una mayor implicación familiar en los estudios del hijo/a, se promueven en los/as adolescentes unas actitudes menos positivas hacia la transgresión de normas derivadas de la autoridad institucional, actuando dicha implicación como factor de protección contra las actitudes transgresoras.

Respecto al profesorado, su percepción de mayor apoyo y ayuda de los centros escolares a las familias predice en los/as adolescentes unas actitudes más positivas hacia la autoridad institucional. De modo, que también el hecho de que el profesorado perciba esta colaboración del centro con las familias resulta un factor protector contra las actitudes transgresoras de los adolescentes. Resulta inquietante, sin embargo, que del lado del profesorado cuanto más percibe que la familia se implica en los estudios del hijo/a menos positiva es la actitud de los/as adolescentes hacia la autoridad institucional. Como se ha señalado, en este estudio la visión del profesorado difiere de la de las familias, señalando una percepción menos positiva de esta implicación en las tareas escolares de sus hijos/as. Es posible que el profesorado perciba que las familias atienden a los estudios de sus hijos/as cuando éstos tienen problemas, lo que predice la actitud positiva hacia la transgresión contra las normas de la autoridad institucional que reportan los hijos/as. A menudo son las familias cuyos/as hijos/as tienen problemáticas académicas las que, según la percepción del profesorado, solicitan con mayor frecuencia ayuda a los docentes (Giró et al., 2014). Por otra parte, como se ha comentado en otro momento, el mismo profesorado considera que, en ocasiones, tiende a tener actitudes poco favorecedoras de la participación de los padres en los centros (Consejo Escolar del Estado, 2015). A su vez, los problemas en los estudios pueden ir aparejados con episodios de transgresión de las normas escolares (Cava, Musitu y Murgui, 2006), y el cuestionamiento de su importancia (Smith, 2016; VegaGea, Ortega-Ruiz y Sánchez, 2016), que, en definitiva, pueden ir vinculadas con las actitudes positivas hacia la transgresión de otras normas sociales (Emler, Ohana y Moscovici, 1987; Estévez y Emler, 2009).

Así pues, se puede considerar que la relación que se establece entre el centro escolar y las familias, cuando se basa en la colaboración conjunta y el logro de metas comunes (Blanco y Martínez-González, 2017), actúa como un factor de protección ante las conductas transgresoras de los/as adolescentes (Estévez et al., 2007), promoviendo modelos positivos (Williams y Bryan, 2013; Williams y Portman, 2014). Por ello, se espera que los centros dinamicen las relaciones con la familia, facilitando así la promoción de conductas ajustadas socialmente en la adolescencia (Povedano et al., 2015; Steffgen et al, 2013). Es importante que los profesores promuevan el que los padres se impliquen en los estudios de los hijos/as no solo cuando haya problemas sino en todos los casos y que el colegio espere y fomente este tipo de apoyo familiar más preventivo.

Respecto a las limitaciones del estudio, sería conveniente incluir información cualitativa que proporcione testimonios directos sobre los respectivos puntos de vista de los participantes. Sin embargo, el hecho de contar con reportes de la familia, los profesores y del alumnado, interrelacionados entre sí, ha permitido profundizar en la temática de análisis desde sus diferentes perspectivas. De este modo, el estudio realizado aporta claves importantes en el estudio de la actitud positiva ante la autoridad institucional durante la etapa de la adolescencia, al señalar la importancia de la colaboración entre el contexto escolar y familiar en su promoción. Concretamente, se han identificado algunos factores vinculados con la percepción de las familias sobre la colaboración con el profesado o su grado de implicación de los estudios de sus hijos/as, así como la percepción del profesorado sobre la ayuda que el centro escolar presta a la familia, como elementos que promueven en los/as adolescentes conductas ajustadas socialmente ante la autoridad institucional; estos aspectos actúan como factores de protección que promueven el bienestar subjetivo en los/as adolescentes y el sentimiento de respeto hacia la autoridad institucional.

Las propuestas de intervención se centran en la prevención primaria actuando el centro escolar, y de manera específica el profesorado, comoagente que puede promover la participación de la familia, para prevenir el desarrollo de conductas transgresoras con las normas sociales en los/as adolescentes en otros contextos más allá de la escuela, y con ello, favorecer su desarrollo cívico y su ajuste personal. Resulta necesario crear canales de comunicación entre ambos contextos, eliminado las barreras que dificultan o impiden la colaboración, y aunando esfuerzos basados en la confianza, el respeto y el reconocimiento de las aportaciones mutuas, porque ello, repercutirá positivamente en los/as adolescentes. Así mismo, resulta de interés fomentar la implicación de la figura del padre en el proceso de colaboración con el centro escolar.

\section{Referencias bibliográficas}

Álvarez, L., y Martínez, R.A. (2017). Review on School and Family Partnership. Shared Responsibility as Quality Indicator. In González, J.L., Bernardo, A., Núñez, J.C., and Rodríguez, C. (2017). Factors Affecting Academic Performance (121-140). New York: Nova Science Publisher.

Batle, M., Hernández, M., y Mir, M. (2009). Contextos de colaboración familia-escuela durante la primera infancia. 
Revista Electrónica de Investigación e Innovación Educativa y Socioeducativa, 1(1), 45-68.

Burt, M., Resnick, G., y Novick, E. (1998). Building supportive communities for at-risk adolescents. Washington DC: American Psychological Association.

Castro, A. y García-Ruiz, R. (2013). La visión del profesorado de infantil y primaria de Cantabria sobre la participación y las relaciones interpersonales entre los miembros de la comunidad escolar. Aula Abierta, 41(1), 73-84

Cava, M. J., Musitu, G., y Murgui, S. (2006). Familia y violencia escolar: el rol mediador de la autoestima y la actitud hacia la autoridad institucional. Psicothema, 18, 367-373.

Consejo Escolar del Estado (2015). Las relaciones entre familia y escuela. Experiencias y buenas prácticas. XXIII Encuentro de consejos escolares autonómicos y del estado. Madrid: Secretaría General Técnica.

Defensor del Pueblo (2007). Violencia escolar: el maltrato entre iguales en la ESO 1999-2006. Madrid: Defensor del Pueblo.

Dekovic, M., Wissink, I.B., y Meijer, A.M. (2004). The role of family and peer relations in adolescent antisocial behaviour: comparison of four ethnic groups. Journal of Adolescence, 27, 497-514.

Diener, E., y Fujita, F., (1995). Resources, Personal striving, and subjective well-being: A nomothetic and idiographic approach. Journal of Personality and Social Psychology, 68(5), $926-935$.

Domínguez, J., López, A., y Álvarez, E. (2015). Implicación de variables sociales y educativas en la conducta asertiva adolescente. Aula Abierta, 43, 26-31.

Estévez, E., y Emler, N. (2009). Individual differences in attitude to school and social reputation among peers: Implications for behavioral adjustment in educational settings. En J. E. Larson (Ed.), Educational Psychology: Cognition and Learning, Individual Differences and Motivation (pp. 342-375). New York: Nova Science Publishers.

Estévez, E., Jiménez, T., Moreno, D., y Musitu, G. (2013). From victim to aggressor: An analysis of the relationship between victimization and violent behavior at school. Spanish Journal of Psychology, 16, 1-13.

Feito, R. (2014). Treinta años de consejos escolares. La participación de los padres y de las madres en el control y gestión de los centros sostenidos con fondos públicos en España. Profesorado, Revista de Currículum y Formación del Profesorado, 18(2), 51-67.

Frías del Val, A.S. (2014). Evolución del marco normativo español sobre la participación de las familias. Contexto histórico. Ministerio de Educación, Cultura y Deporte (Ed.), La participación de las familias en la educación escolar (pp. 5780).

Gârțu, M. L. (2017). The School-Family Educational Partnership. Journal of Pedagogy, 2, 107-123.

Giró, J., Mata, A., Vallespir, J., y Vigo, B. (2014). Familias y escuelas: los diferentes discursos sobre la participación. Equidad International Welfare Policies and Social Work Journal, 2, 65-90.

Jeynes, W. (2007). The relationship between parental involvement and urban secondary school student academic achievement: A meta-analysis. Urban Education, 42, 82-110.

López-Larrosa, S., y Dubra, M. (2010). Las relaciones familiaescuela desde la perspectiva de los adolescentes. Revista galego-portuguesa de psicoloxía e educación: revista de estudios e investigación en psicología y educación, 18(2), 181-188.

Martínez-González, R. A. (1996). Familia y educación. Oviedo: Universidad de Oviedo.
Martínez, R. A. y Álvarez, L. (2005). Fracaso y abandono escolar en educación secundaria obligatoria: implicación de la familia y los centros escolares. Aula Abierta, 85, 127146.

Martínez, R.A., Rodríguez-Ruiz, B., y Gimeno, J.J. (2010). Áreas de cooperación entre los centros docentes y las familias. Estudio de caso. Educatio Siglo XXI, 28(1), 127-156.

Ministerio de Educación, Cultura y Deporte (Ed.) (2014). La participación de las familias en la educación escolar. Madrid: Ministerio de Educación, Cultura y Deporte.

Moncher, F. J., y Miller, G. E. (1999). Nondelinquent youths' stealing behavior and their perceptions of parents, school, and peers. Adolescence, 34, 577-591.

Musitu, G., y Cava, M. J. (2001). La familia y la educación. Barcelona: Octaedro.

Nord, C.W., Brimhall, D., y West, J. (1997). Fathers' Involvement in their Children's Schools. Washington, DC: U.S. Department of Education, National Center for Education Statistics.

Oliva, A., y Parra, A. (2004). Contexto familiar y desarrollo psicológico durante la adolescencia. En E. Arranz (Ed.) Familia y desarrollo psicológico (pp.96-123). Madrid: Pearson Educación.

Pacheco, R., Rodríguez, Mª . R., y García, R. (2013). Impacto de las brechas de género y generacional en la construcción de actitudes en padres y madres frente a las innovaciones coeducativas. Profesorado, Revista de Currículum y Formación del Profesorado, 17(1), 182-200.

Povedano, A., Cava, M. J., Monreal, M. C., Varela, R., y Musitu, G. (2015). Victimization, loneliness, overt and relational violence at the school from a gender perspective. International Journal of Clinical and Health Psychology, 15, 44-51.

Reicher, S., y Emler, N. (1985). Delinquent behaviour and attitudes to formal authority. British Journal of Social Psychology, 3, 161168.

Risko, V., y Walker-Dalhouse, D. (2009). Parents and teachers: Talking with or past one another. The Reading Teacher, 62(5), 442-444.

Rodrigo, M.J., Máiquez, M.L., Martín, J.C., Byrne, S., y Rodríguez, B. (2015). Manual práctico de parentalidad positiva. Madrid: Síntesis.

Rodrigo, M. J., García, M., Máiquez, M. L., Rodríguez-Ruiz, B., y Padrón, I. (2008). Estrategias y metas en la resolución de conflictos cotidianos entre adolescentes, padres y madres. Infancia y Aprendizaje, 3(3), 347-362.

Rodríguez-Ruiz, B. (2012). Resolución de conflictos y desarrollo positivo en la adolescencia: Efecto de la coherencia familia-escuela. La Laguna: Universidad de La Laguna.

Ruiz-Juan, F., y Ruiz-Risueño, J. (2011). Variables predictoras de consumo de alcohol entre adolescentes españoles. Anales de Psicología, 27(2), 350- 359.

Sarramona, J. y Rodríguez, T. (2010). Participación y calidad de la educación. Aula Abierta, 38(1), 3-14.

Smith, P.K., y Brain, P. (2000) Bullying in schools: Lessons from two decades of research. Aggressive Behavior, 26, 1-9.

Trianes, M.V. (2000). La violencia en contextos escolares. Málaga: Aljibe.

Valdés, Á. A., Martín, M., y Sánchez, P. A. (2009). Participación de los padres de alumnos de educación primaria en las actividades académicas de sus hijos. Revista Electrónica de Investigación Educativa, 11(1).

Vallespir, J.; Rincón, J. C., y Morey, M. (2016). La participación de las familias en el Consejo Escolar y la formación del profesorado. Revista Electrónica Interuniversitaria de Formación del Profesorado, 19(1) 31-45. 
Vega-Gea, E. M., Ortega-Ruiz, R., y Sánchez, V. (2016). Peer sexual harassment in adolescence: Dimensions of the sexual harassment survey in boys and girls. International Journal of Clinical and Health Psychology, 16, 47-57.

Williams, J., y Bryan, J. (2013). Overcoming adversity: highachieving African American youth's perspectives on educational resilience. Journal of Counseling y Development, 91(3), 291-300. 